\title{
Silencing FAT10 inhibits metastasis of osteosarcoma
}

\author{
CHENGBIN MA $^{1}$, ZHIYU ZHANG ${ }^{2}$, YAN CUI ${ }^{3}$, HONGMOU YUAN ${ }^{4}$ and FENG WANG ${ }^{1}$ \\ Departments of ${ }^{1}$ Spine Surgery, ${ }^{2}$ Joint and Oncological Surgery, ${ }^{3}$ Joint Surgery, ${ }^{4}$ Traumatology, Department of Orthopedics, \\ The Fourth Affiliated Hospital, China Medical University, Huanggu, Shenyang, Liaoning 110033, P.R. China
}

Received March 3, 2016; Accepted April 18, 2016

DOI: 10.3892/ijo.2016.3549

\begin{abstract}
Metastasis is the main challenge of osteosarcoma treatment. Herein, we first reveal the oncogenic role of FAT10 in metastasis of osteosarcoma. FAT10 was upregulated in osteosarcoma, especially in metastatic osteosarcoma. High level of FAT10 was associated with poorer prognosis of osteosarcoma patients. Moreover, Transwell and Matrigel assays revealed that silencing FAT10 significantly inhibited the invasive and migratory abilities of osteosarcoma cells. Metastasis assay in vivo showed that silencing FAT10 decreased the number of mice with distant metastasis. We also found that FAT10 may act its oncogenic functions through regulating HOXB9. Collectively, the results suggested that FAT10 may be a novel therapeutic target for osteosarcoma patients.
\end{abstract}

\section{Introduction}

Osteosarcoma (OS), the frequent sites of which are the femur, the tibia and the humerus, is the most common primary malignant bone tumor in the world, representing approximately $56 \%$ of all bone cancers (1). By combining surgery with multi-agent chemotherapy, the 5-year cumulative survival rate of primary OS has significantly improved to $60-90 \%$ in the past three decades $(2,3)$. Unfortunately, approximately $80 \%$ of patients eventually developed metastasis following surgical treatment and pulmonary metastasis in OS patients is a major cause of fatal outcome (4,5). Therefore, it is crucical to identify effector molecules and signaling pathways which exhibit a close relationship with tumor progression and metastasis. Novel findings may improve the existing OS treatment.

HLA-F locus adjacent transcript 10 (FAT10) is a member of the ubiquitin-like protein family, which covalently modifies target substrates by binding via their C-termini containing conserved Gly-Gly motifs $(6,7)$ and is well known as a signal

Correspondence to: Dr Zhiyu Zhang, Department of Joint and Oncological Surgery, Department of Orthopedics, The Fourth Affiliated Hospital, China Medical University, 4 Chongshan Dong Road, Huanggu, Shenyang, Liaoning 110033, P.R. China

E-mail: zhangzy0033@sina.com

Key words: FAT10, HOXB9, osteosarcoma, $\beta$-catenin for proteasomal degradation (8). FAT10 has been confirmed to be related with cell growth and survival; the ectopic expression of FAT10 could induce abnormal alterations in the cell cycle, apoptosis, and immune response $(7,9,10)$. Recently, the role of FAT10 in malignant tumors has been widely studied. Overexpression of FAT10 was examined in various cancers, such as gastrointestinal cancer (11), hepatocellular carcinoma (HCC) $(12,13)$, pancreatic ductal adenocarcinoma (14), and human glioma (15). FAT10 has been proved to significantly promote invasion and metastasis in mutliple cancers $(11,14,16)$. However, the role of FAT10 in OS was not illuminated. Homeobox B9 (HOXB9), a member of the class I homeobox (HOX) genes, played a critical role in embryonic segmentation and limb patterning (17). Recently, biologic function of HOXB9 in tumor metastasis has been confirmed. It has been reported that the overexpression of HOXB9 in breast cancer could increase angiogenesis and distal metastasis, and the knockdown of HOXB9 significantly decreased the ability of lung cancer cells to form bone and brain metastases $(18,19)$. Moreover, silencing FAT10 could inhibit HOXB9 expression in HCC cells. Thus, we presumed that HOXB9 may be the regulatory target of FAT10 in invasion and metastasis.

In this study, we found that FAT10 was aberrantly upregulated in OS, especially in metastatic OS. High expression of FAT10 was correlated with poor prognosis of OS patients. Furthermore, silencing FAT10 significantly inhibit invasion and metastasis of OS cells by regulating HOXB9.

\section{Materials and methods}

Clinical specimens and cell culture. This study was conducted with the approval of Institutional Ethical Review Board of the Fourth Affiliated Hospital, China Medical University. A total of 65 pairs of specimens and their adjacent tissue samples frozen in liquid nitrogen were obtained from the Fourth Affiliated Hospital. No patients had received any antitumor treatments before biopsy. All cell lines are cultured in adaptive culture medium according to ATCC and cultured at $37^{\circ} \mathrm{C}$ in $5 \% \mathrm{CO}_{2}$.

Establishment of cell lines. Human shRNA of FAT10 were cloned into pSuper-puro vector. Retrovirus supernatants were pSuper and pSuper-sh. PKM2 were produced in Phoenix packaging cells. We respectively transfected the indicated cell lines with these different viral supernatant containing $4 \mu \mathrm{g}$ / $\mathrm{ml}$ polybrene (Sigma). Then cells were selected by puromycin (2 $\mu \mathrm{g} / \mathrm{ml})$. The methods were as described (20). 
Table I. Association of FAT10 expression in human osteosarcoma tissues with clinicopathological features.

\begin{tabular}{|c|c|c|c|c|}
\hline \multirow[b]{2}{*}{ Clinicopathological features } & \multirow[b]{2}{*}{ No. of patients } & \multicolumn{2}{|c|}{ FAT10 expression } & \multirow[b]{2}{*}{ P-value } \\
\hline & & Low $(\mathrm{n}, \%)$ & $\operatorname{High}(\mathrm{n}, \%)$ & \\
\hline \multicolumn{5}{|l|}{ Gender } \\
\hline Male & 35 & $16(45 \%)$ & $19(55 \%)$ & NS \\
\hline Female & 30 & $17(57 \%)$ & $13(43 \%)$ & \\
\hline \multicolumn{5}{|l|}{ Age } \\
\hline$<25$ years & 26 & $14(54 \%)$ & $12(46 \%)$ & NS \\
\hline$\geq 25$ years & 39 & $18(55 \%)$ & $19(45 \%)$ & \\
\hline \multicolumn{5}{|l|}{ Tumor size } \\
\hline$>8 \mathrm{~cm}$ & 34 & $11(32 \%)$ & $23(68 \%)$ & 0.012 \\
\hline$\leq 8 \mathrm{~cm}$ & 31 & $17(55 \%)$ & $14(45 \%)$ & \\
\hline \multicolumn{5}{|c|}{ Serum level of alkaline phosphatase } \\
\hline Elevated & 49 & $31(63 \%)$ & $18(37 \%)$ & NS \\
\hline Normal & 16 & $8(50 \%)$ & $8(50 \%)$ & \\
\hline \multicolumn{5}{|l|}{ Anatomic location } \\
\hline Tibia/femur & 44 & $21(48 \%)$ & $23(52 \%)$ & NS \\
\hline Elsewhere & 21 & $11(52 \%)$ & $10(48 \%)$ & \\
\hline \multicolumn{5}{|l|}{ Distant metastasis } \\
\hline Absent & 48 & $32(67 \%)$ & $16(33 \%)$ & $<0.001$ \\
\hline Present & 17 & $5(29 \%)$ & $12(71 \%)$ & \\
\hline \multicolumn{5}{|l|}{ Response to chemotherapy } \\
\hline Good & 24 & $18(75 \%)$ & $6(25 \%)$ & $<0.001$ \\
\hline Poor & 41 & $13(32 \%)$ & $28(68 \%)$ & \\
\hline \multicolumn{5}{|l|}{ Clinical stage } \\
\hline IIA & 29 & $23(79 \%)$ & $6(21 \%)$ & $<0.001$ \\
\hline IIB/III & 36 & $7(19 \%)$ & $29(81 \%)$ & \\
\hline \multicolumn{5}{|c|}{ Serum level of lactate dehydrogenase } \\
\hline Elevated & 43 & $25(58 \%)$ & $17(42 \%)$ & NS \\
\hline Normal & 22 & $10(45 \%)$ & $12(55 \%)$ & \\
\hline
\end{tabular}

RNA extraction, reverse transcription, and real-time RT-PCR. Total RNA was extracted from freshly-frozen samples or cells with TRIzol reagent (Invitrogen). Total RNA was reversetranscribed with First Strand cDNA Synthesis kit (Invitrogen). Real-time PCR reactions were conducted using Platinum SYBR Green qPCR SuperMix-UDG reagents (Invitrogen) on the PRISM 7900HT system (Applied Biosystems). All reactions were done in triplicate and reactions without reverse transcriptase were used as negative controls. The GAPDH were used as the endogenous controls and the $2^{-\Delta \Delta C T}$ equation was used to calculate the relative expression levels.

Western blot analysis. Western blot analysis was conducted using anti-FAT10, anti- $\beta$-catenin and anti- $\beta$-actin. All antibodies were purchased from Cell Signaling Technology.

Cell migration and invasion assays. The effects of FAT10 or HOXB9 expression on cell migration and invasion were assessed using the Transwell and Matrigel assays as previously described (21).

Immunofluorescence. Cells silencing FAT10 were cultured on glass coverslips (NEST, 801007) in 24-well plate and fixed by $4 \%$ paraformaldehyde for $15 \mathrm{~min}$ at $50-60 \%$ density followed by washing in PBS. After blocking in goat serum (1:10 in PBS) for $30 \mathrm{~min}$, the coverslips were incubated with primary antibodies (diluted in primary stain diluting buffer, Beyotime, P0103) overnight at $4^{\circ} \mathrm{C}$ and secondary antibodies $1 \mathrm{~h}$ at $37^{\circ} \mathrm{C}$. Nuclei were visualized by 4',6-diamidine-2-phenylindole staining (DAPI, Solarbio, D8200). The coverslips touched face down a drop of Anti-fade Mounting Medium (Beyotime, P0126) on a slide and the fluorescence was captured by laser scanning confocal microscopy.

In vivo metastasis. Nude mice were purchased from Shanghai Slac Laboratory Animal Co. Ltd. and maintained 


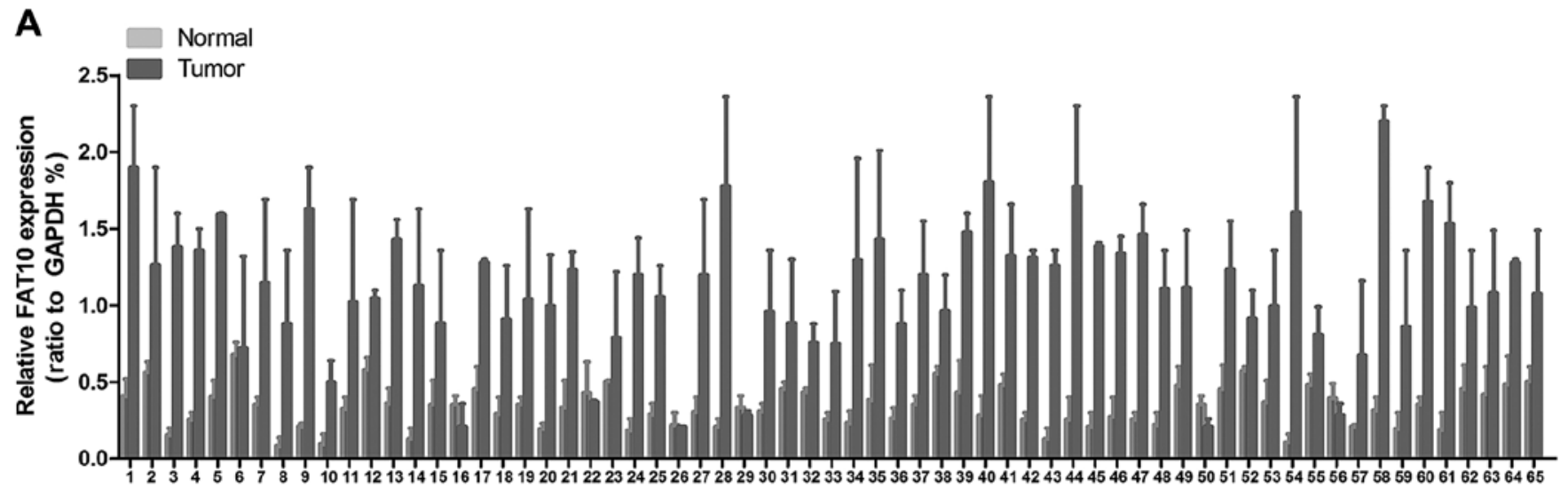

B

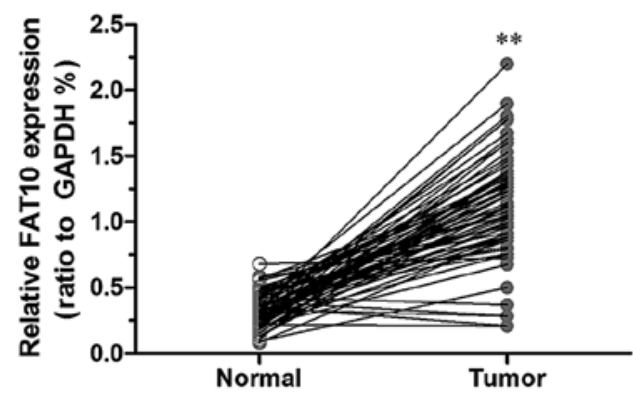

D

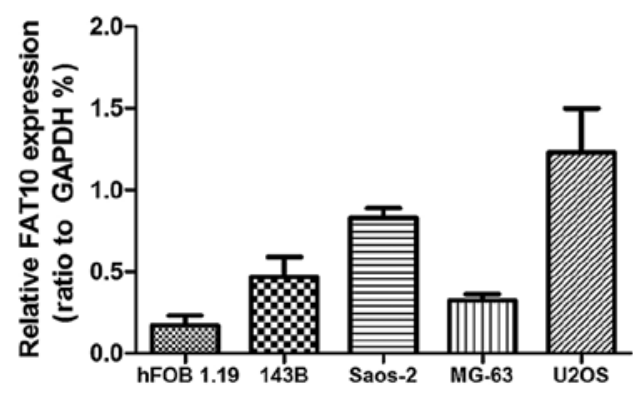

C

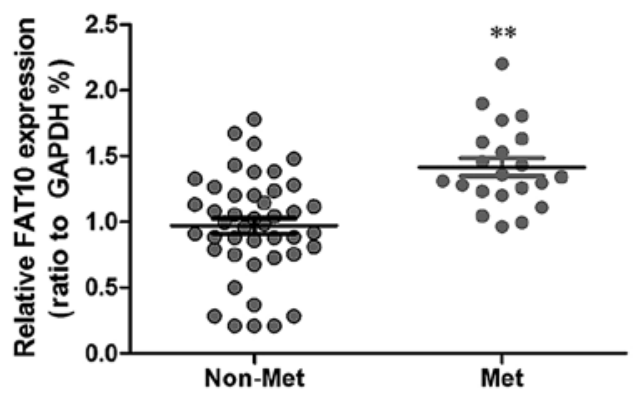

$\mathbf{E}$

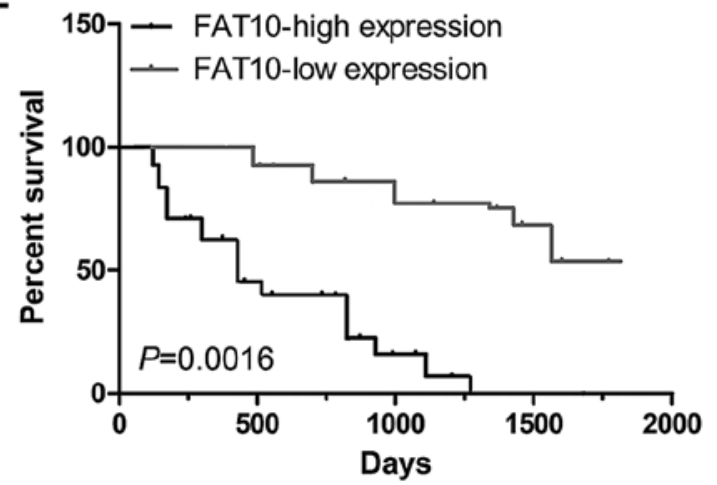

Figure 1. Elevated FAT10 levels were examined in OS and related with metastasis. (A) Quantification of FAT10 RNA in human osteosarcoma (Tumor) and adjacent tissues (Normal). (B) Quantitative analysis of FAT10 levels in human osteosarcoma (Tumor) and adjacent tissues (Normal). (C) Quantitative analysis of FAT10 levels in non-metastatic OS (No-met) and metastatic OS tissues (Met). (D) Quantification of RNA levels of FAT10 in OS cell lines. (E) Correlation analysis of expression data and patient survival data from 65 OS patients collected from the Fourth Affiliated Hospital showing that FAT10 levels are a risk indicator for survival. ${ }^{* *} \mathrm{P}<0.01$ based on the Student's t-test. Error bars, SD.

in microisolator cages. All animals were used in accordance with institutional guidelines and the current experiments were approved by the Use Committee for Animal Care. For metastasis assays, cells were resuspended in PBS at a concentration of $3 \times 10^{7}$ cells $/ \mathrm{ml}$. Cell suspension $(0.1 \mathrm{ml})$ was injected into tail veins of nude mice. All of the mice were sacrificed by $\mathrm{CO}_{2}$ 8 weeks after inoculation.

Statistical analysis. Statistical analysis data are described as the mean \pm SD. Survival percent was estimated using the Kaplan-Meier method. The relationship between survival period and each of the variables was analyzed using the log-rank test for categorical variables. Comparisons between different groups were undertaken using the Student's two-tailed t-test. The criterion of statistical significance was $\mathrm{P}<0.05$. Statistical analysis was done with SPSS/Win11.0 software (SPSS Inc.).

\section{Results}

Ectopic expression of FAT10 was correlated with prognosis of OS patients. In order to confirm whether FAT10 was upregulated in OS, we first examined expression levels of FAT10 in OS by qRT-PCR. As shown in Fig. 1A and B, higher expression level of FAT10 was examined in $90.7 \%$ OS samples which suggested that FAT10 was correlated with the progression of OS. As show in the report, metastasis has been proved to be the key cause of death in cancers. To further confirm the role of FAT10 in OS, we studied the relationship between FAT10 and metastasis. We first found that FAT10 was significantly upregulated in metastatic OS (Fig. 1C) as well as invasive OS cell lines (Fig. 1D). Moreover, the analysis of clinical features of OS patients revealed that higher expression of FAT10 suggested more distant metastasis (Table I). 
A

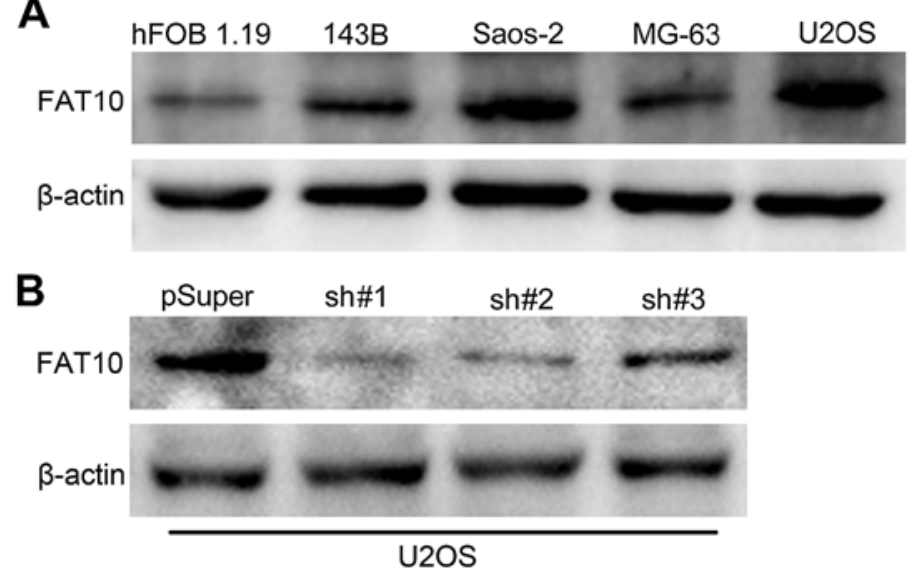

D

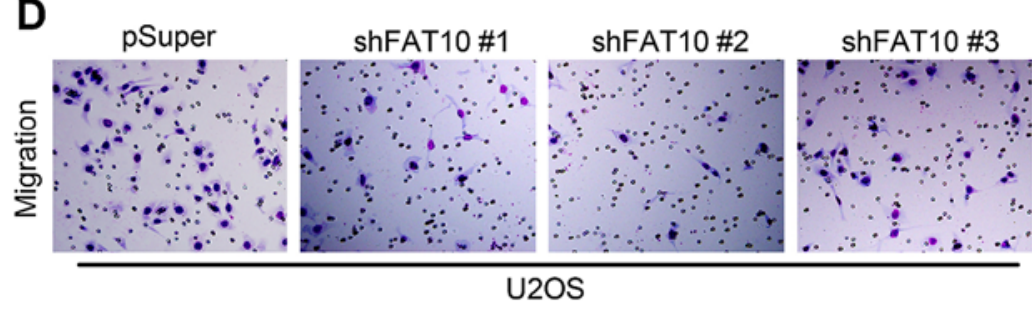

E

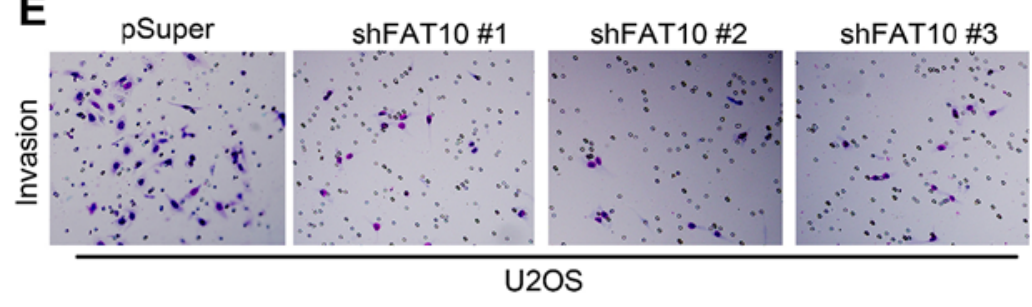

C
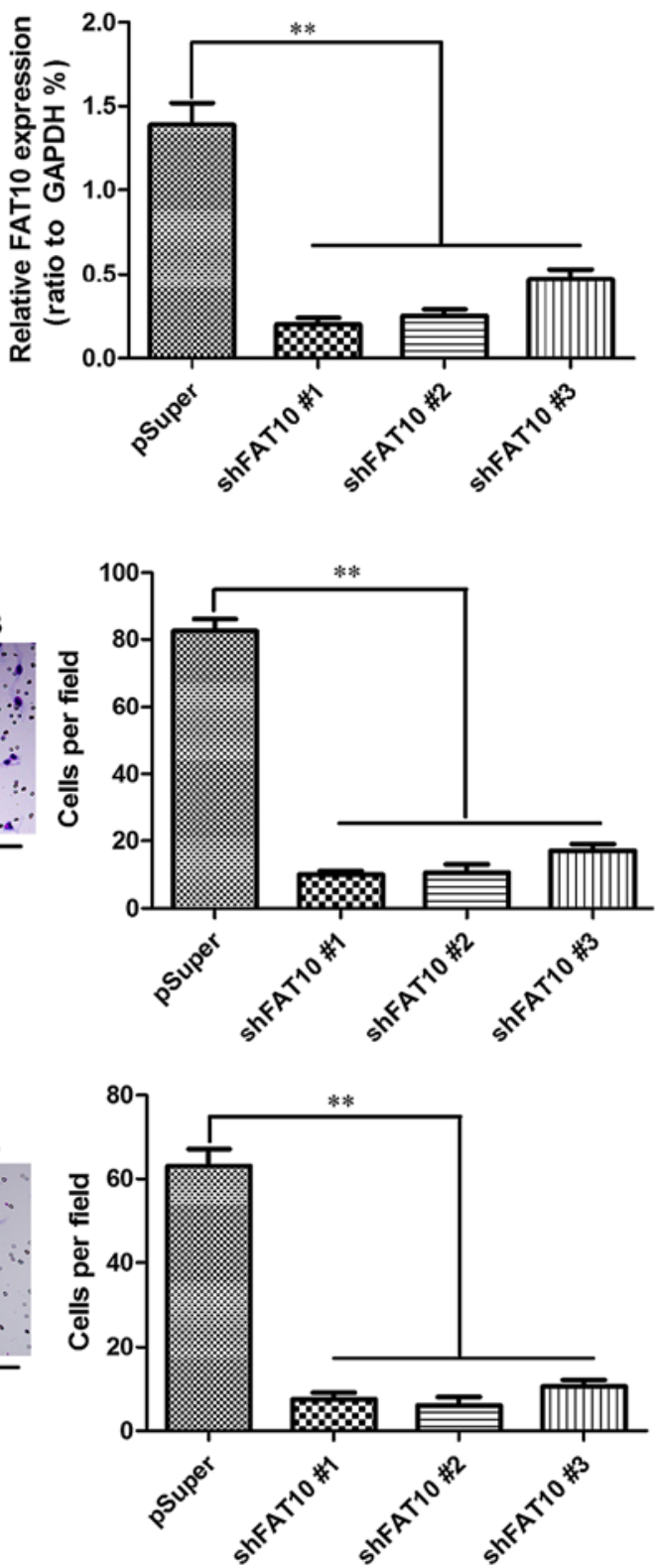

Figure 2. Silencing FAT10 inhibits migration and invasion of OS cells. (A) Western blot of FAT10 in OS cell lines. (B) Western blot for the expression levels of FAT10 in U2OS with silent FAT10 and empty vector pSuper. (C) Quantification of RNA levels of FAT10 in U2OS with silent FAT10 and empty vector pSuper. (D) U2OS with silent FAT10 possessed less migration ability in Transwell assay. (E) U2OS with silent FAT10 possessed less invasion ability in Matrigel assay. ${ }^{* *} \mathrm{P}<0.01$ based on the Student's t-test. Error bars, SD.

These data suggested that FAT10 promoted the metastasis of OS.

Metastasis is closely related with the prognosis of cancer patients. To evaluate whether FAT10 is related with prognosis of OS patients, we carried out bioinformatics analysis of the dataset. As shown in Fig. 1E, patients with higher FAT10 mRNA level in OS tissues had poorer survival than those with lower FAT10 expression level suggesting that FAT10 expression significantly correlated with the prognosis of OS patients.

Silencing FAT1O inhibits migration and invasion of OS cells. To further confirm the role of FAT10 in metastasis, we first examined the expression levels of FAT10 in several OS cell lines by western blotting (Fig. 2A) and then we silenced the expression of FAT10 in U2OS with high expression of FAT10 by shRNA. The subsequent levels of FAT10 were examined by western blotting (Fig. 2B) and qRT-PCR (Fig. 2C). We then evaluated the effects of FAT10 on migration and invasion of OS cells. Transwell assay revealed that silencing FAT10 significantly inhibit the numbers of U2OS cells migrated through the membrane to the bottom of the aperture (Fig. 2D). Moreover, Matrigel assay was used to evaluate the invasive potential of OS cells with altered FAT10 expression. As shown Fig. 2E, silencing FAT10 decreased the numbers of invaded cells. In order to further confirm the role of FAT10 in migration and invasion, we overexpressed FAT10 in MG-63 cells. The expression of FAT10 was confirmed by western blotting (Fig. 3A) 

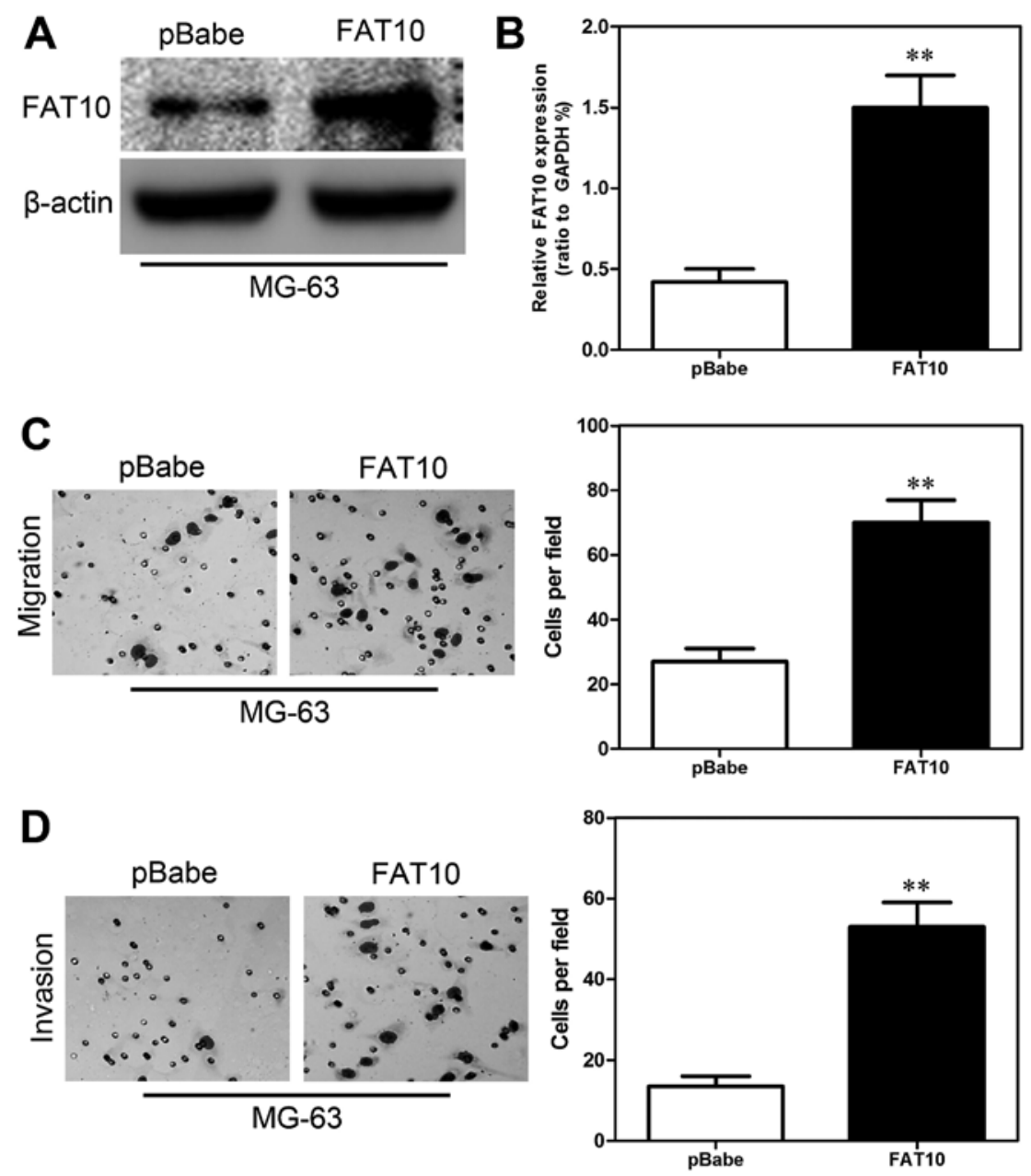

Figure 3. FAT10 promotes migration and invasion of OS cells. (A) Western blot of the expression levels of FAT10 in MG-63 cells with overexpression of FAT10. (B) qRT-PCR of the expression levels of FAT10 in MG-63 cell with overexpression of FAT10. (C) Transwell assay for the MG-63 cells with overexpression of FAT10. (D) Matrigel assay for the MG-63 cells with overexpression of FAT10. ${ }^{* *} \mathrm{P}<0.01$ based on the Student's t-test. Error bars, SD.

A

\begin{tabular}{lc}
\hline U2OS & $\begin{array}{c}\text { Number of mice } \\
\text { with distant metastasis }\end{array}$ \\
\hline pSuper & $8 / 10$ \\
shFAT10 \#1 & $2 / 10$ \\
\hline
\end{tabular}

B

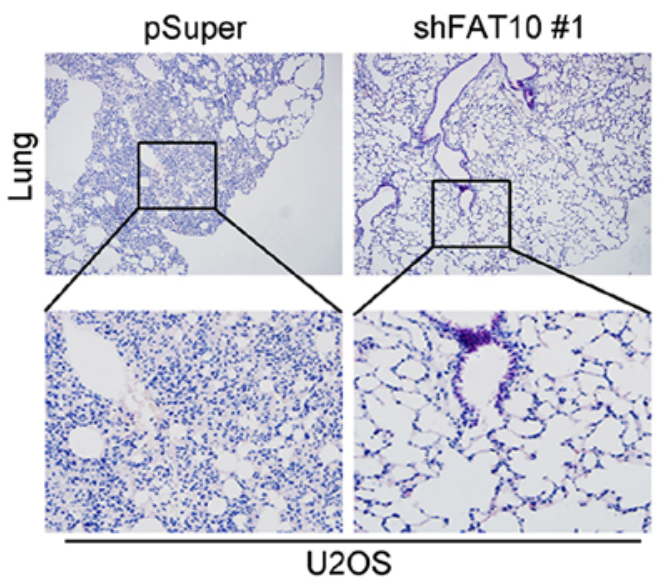

C

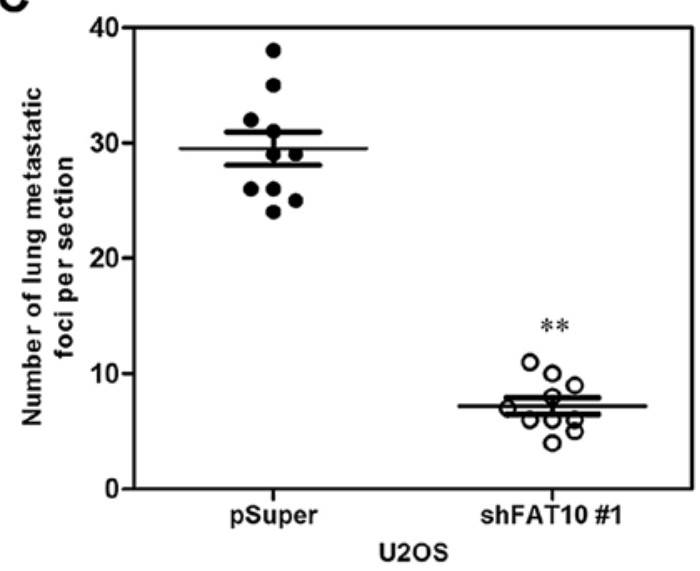

Figure 4. Silencing FAT10 inhibits metastasis of OS cells in vivo. (A) The total numbers of mice with distant metastasis at 8 weeks after injection of U2OS cells with silent expression of FAT10 into the tail vein. (B) The numbers of metastatic foci per section in lung of individual mouse with injection of U2OS cells with silent FAT10. (C) Analysis of the numbers of metastatic foci per section in the lung. ${ }^{* * *} \mathrm{P}<0.01$ based on the Student's t-test. Error bars, SD. 
A
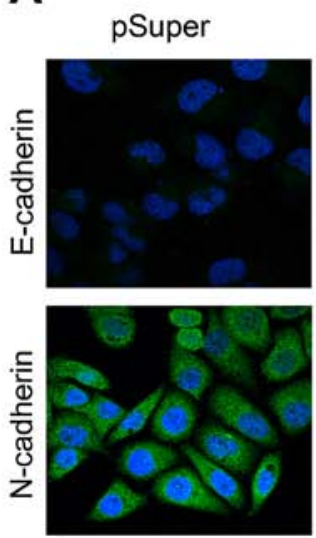

shFAT10
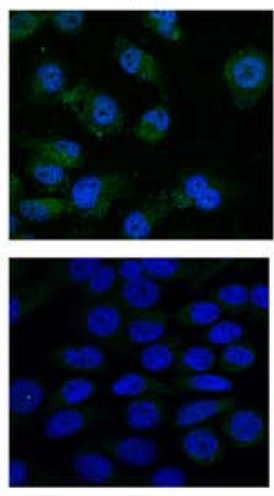

\#2
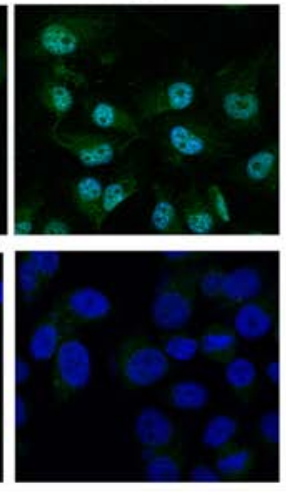

U2OS

B
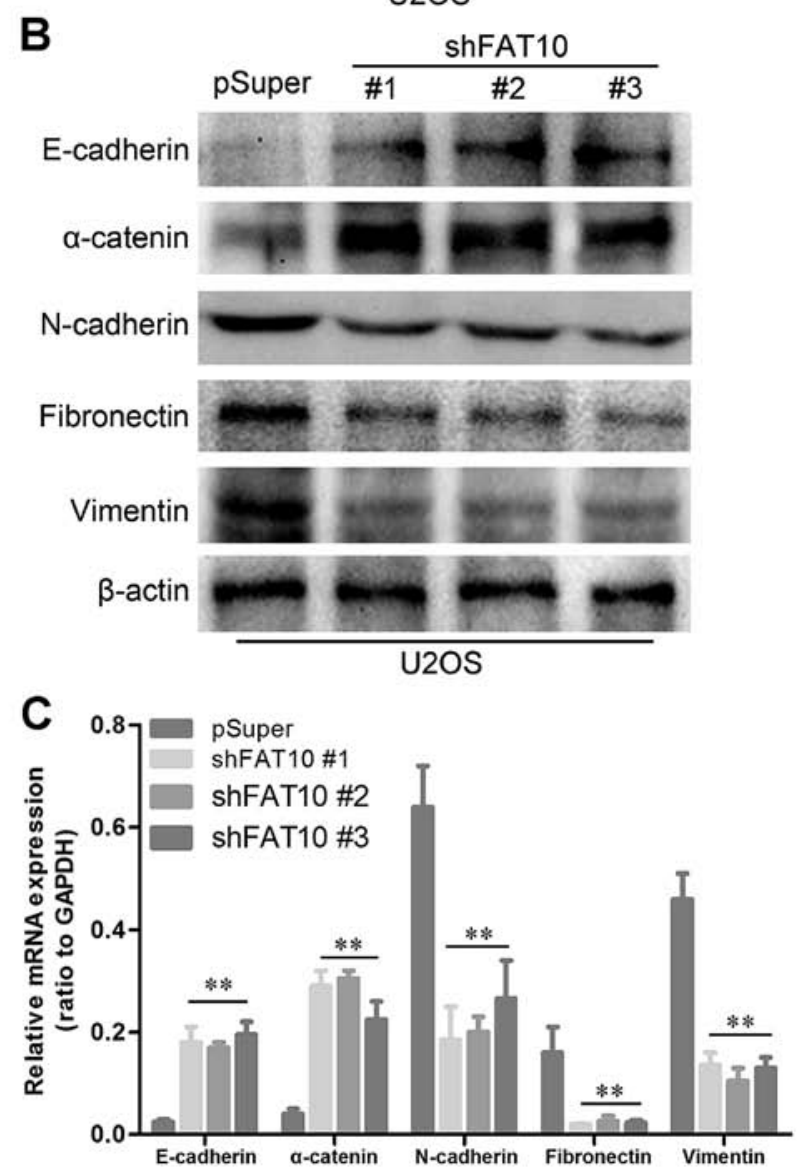

Figure 5. FAT10 induces EMT of OS cells. (A) Immunofluorescence images of EMT markers in U2OS cells with silent FAT10. (B) Western blot analysis of the expression of the epithelial cell marker E-cadherin and $\alpha$-catenin, and the mesenchymal cell markers vimentin, fibronectin and $\mathrm{N}$-cadherin in U2OS cells with silent FAT10. (C) qRT-PCR analysis of the expression of the epithelial cell marker E-cadherin and $\alpha$-catenin, and the mesenchymal cell markers vimentin, fibronectin and N-cadherin in U2OS cells with silent FAT10. ${ }^{* *} \mathrm{P}<0.01$ based on the Student's t-test. Error bars, SD.

and qRT-PCR (Fig. 3B). Then we examined the migratory and invasive abilities of MG-63-FAT10 cells. Transwell assay revealed that overexpression of FAT10 significantly increased the number of migrated cells (Fig. 3C) and Matrigel assay also showed similar results (Fig. 3D). These results revealed that FAT10 was essential for the migration and invasion of OS cells.

Silencing FAT10 inhibits osteosarcoma cell metastasis in vivo. To examine whether the function of FAT10 in migration and invasion in vitro was relevant to metastasis of OS cells in vivo, U2OS cells with silent FAT10 was inoculated into tail vein of
BALB/C athymic mice. After 8 weeks, we observed that less mice, injected with OS cells with silent FAT10, had distant metastasis (Fig. 4A). In addition, less metastatic foci in lungs were counted in each mouse injected with OS cells with silent FAT10 (Fig. 4B and C).

Collectively, both in vivo and in vitro data strongly showed the biological role of FAT10 as an inducer of metastasis in OS. Silencing FAT10 inhibited EMT of OS cells. Epithelial mesenchymal transformation (EMT) has been proved to be the key step of metastasis. To evaluate the role of FAT10 in EMT, 
A

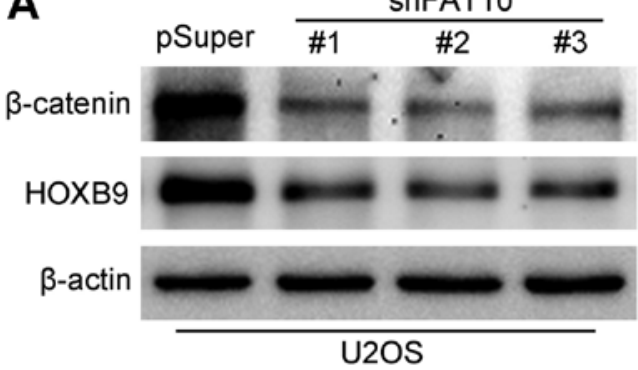

C

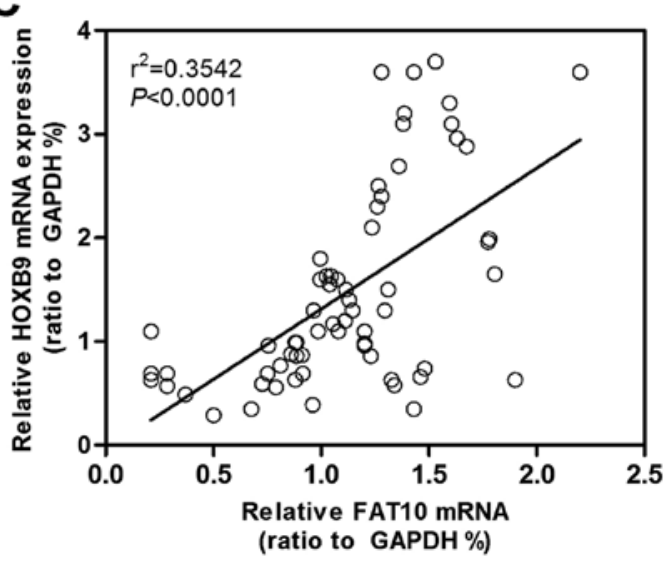

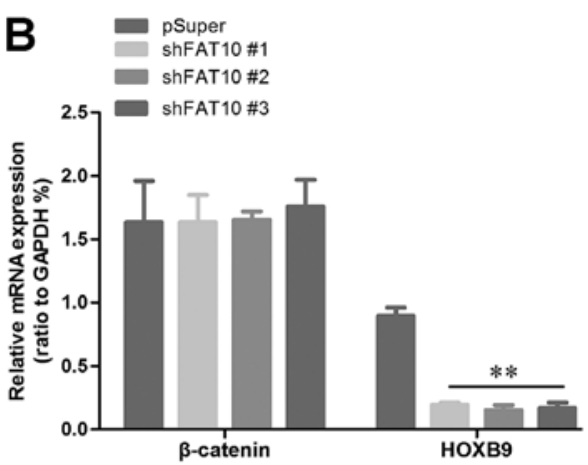
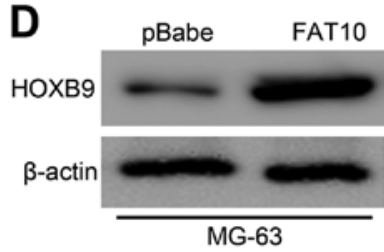

Figure 6. FAT10 regulated $\beta$-catenin. (A) Western blot analysis of $\beta$-catenin and HOXB9 in U2OS cells with silent FAT10. (B) qRT-PCR analysis of $\beta$-catenin and HOXB9 in U2OS cells with silent FAT10. (C) Correlation analysis between FAT10 and HOXB9 levels. (D) Western blot of HOXB9 in MG-63 cells with overexpression of FAT10. ${ }^{* *} \mathrm{P}<0.01$ based on the Student's t-test. Error bars, SD.
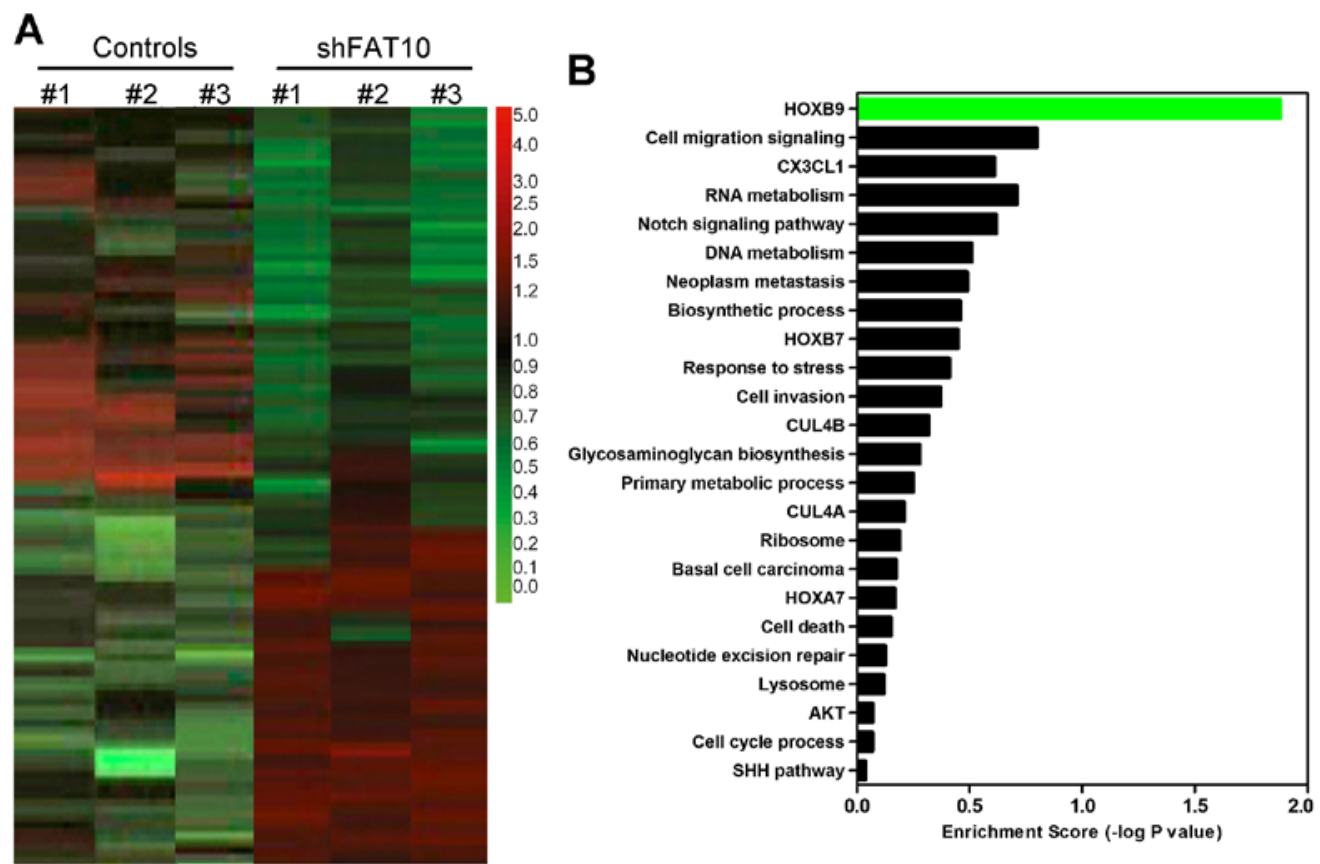

Figure 7. Microarrey of OS cells with silenced FAT10. (A) OS cells with silenced FAT10. (B) Gene enrichment of OS cells with silenced FAT10.

we examined the expression levels of some EMT markers. As shown in Fig. 5A, upregulated E-cadherin and loss of $\mathrm{N}$-cadherin were found in U2OS with silent FTA10 by immunofluorescence. Moreover, western blotting revealed upregulated levels of epithelial markers and loss of mesenchymal markers (Fig. 5B). We also revealed that all the changes of EMT-related proteins were based on the ectopic expressions of EMT-related genes suggesting that FAT10 may regulate the expression of EMT markers at DNA levels (Fig. 5C).

HOXB9 maybe the key mediator of FAT10-induced metastasis of OS patients. HOXB9 has been reported to be a regulatory target of FAT10, here we also found downregulated HOXB9 in U2OS with silent FAT10 (Fig. 6A) while upregulated HOXB9 

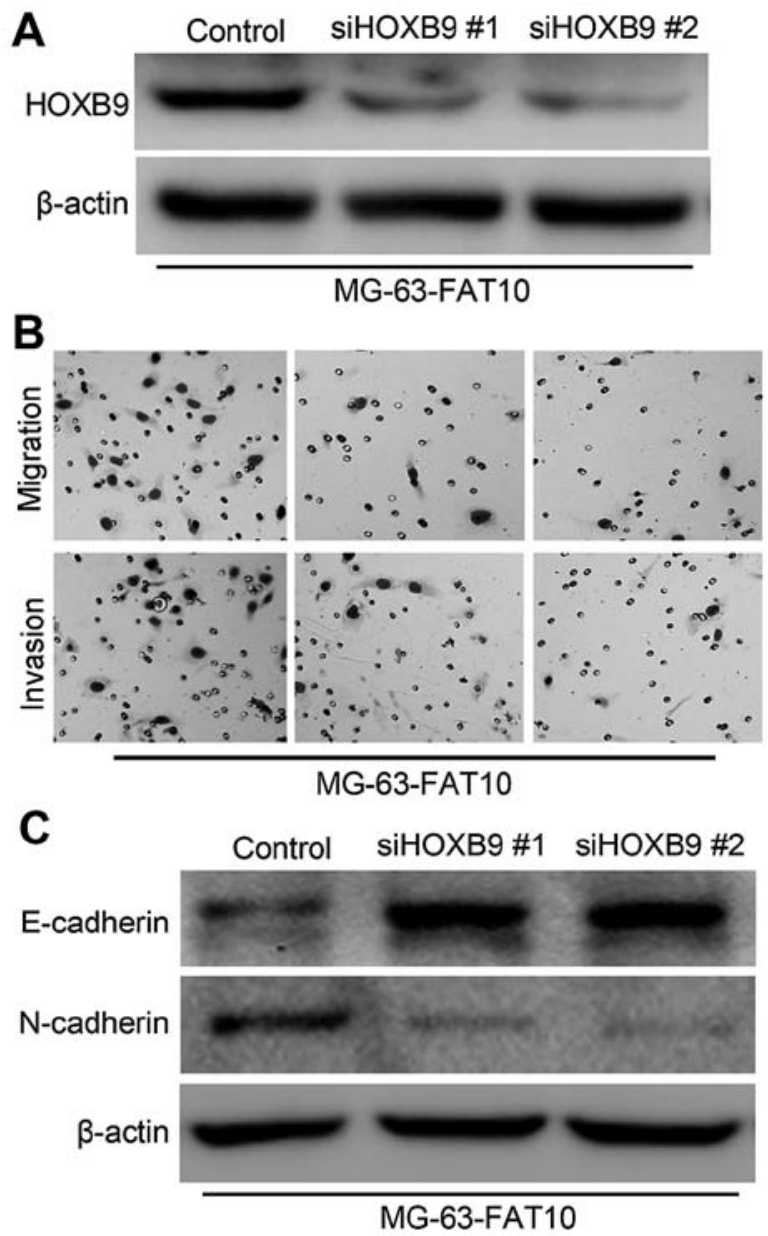

Figure 8. Silencing HOXB9 inhibits the function of FAT10. (A) Western blot of the expression levels of HOXB9 in MG-63 cells with overexpression of FAT10. (B) Transwell and Matrigel assay of MG-63 cells with overexpression of FAT10 and silenced HOXB9. (C) Western blot for the EMT markers of MG-63 cells with overexpression of FAT10 and silenced HOXB9.

was examined in MG-63-FAT10 cells (Fig. 6D) suggesting that FAT10 may regulate expression of HOXB9. Western blotting also revealed that $\beta$-catenin which activated expression of HOXB9 decreased in U2OS-shFAT10 cells. Moreover, we found a decrease in RNA level of HOXB9 but no changes in RNA level of $\beta$-catenin (Fig. 6B) which indicated that posttranscriptional modification occurred in $\beta$-catenin. In order to further confirm the relationship between FAT10 and HOXB9, we performed linear correlated analysis. As shown in Fig. 6C, the expression levels of HOXB9 were positively correlated with the expression levels of FAT10. We confirmed the relationship between FAT10 and HOXB9 by microarray assay. As shown in Fig. 7A, silencing FAT10 induced, respectively, increasing expression (red) and decreasing expression (green) at DNA levels. HOXB9 was significantly inhibited by FAT10 in gene enrichment assay (Fig. 7B). These data suggested that FAT10 regulated HOXB9 possibly through $\beta$-catenin pathway.

In order to confirm the role of HOXB9, we silenced the expression of HOXB9 in MG-63 cells with overexpression of FAT10. The expression levels of HOXB9 were confirmed by western blotting (Fig. 8A). Then we examined the invasiveness and migration MG-63 cells. As shown in Fig. 8B, Transwell and Matrigel assays revealed that silencing HOXB9 signifi- cantly decreased the number of migrated and invaded cells in MG-63-FAT10 cells. We also found that silencing HOXB9 promoted the expression of epithelial marker E-cadherin but inhibited the expression of mesenchymal marker $\mathrm{N}$-cadherin (Fig. 8C). The data above suggested that HOXB9 was essential in the FAT10-induced migration and invasion.

\section{Discussion}

FAT10 is a member of the ubiquitin-like protein family. Herein, we first revealed that FAT10 could induce EMT in OS cells and promoted migration and invasion of OS cells in vitro as well as metastasis in vivo. Moreover, FAT10 was upregulated in OS, especially in metastatic OS and higher expression level of FAT10 was correlated with poorer prognosis of OS patients. In this study, we revealed that FAT10 promoted metastasis of OS possibly by regulating $\beta$-catenin pathway in which HOXB9 played a critical role.

In recent years, FAT10 has been shown to participate in tumor migration and invasion. In a report, FAT10 promoted cells invasion and migration through regulating NF- $\mathrm{\kappa B}-$ CXCR4/7 pathway (22). In another study, FAT10 promoted the invasion of HCC cells via the Akt/GSK3b pathway (23). Moreover, HOXB9 has been shown to be upregulated by FAT10 in invasion and metastasis of hepatocarcinoma cells (16). In this study, we suggested a similar mechanism in which FAT10 promoted OS cell invasion and metastasis by upregulating HOXB9. First, FAT10 inhibition reduced HOXB9 expression and decreased the invasion and metastasis of OS cells in vitro and in vivo. Second, the logistic regression results revealed that co-overexpression of FAT10 and HOXB9. These data suggested a possible mechanism in which FAT10 promoted metastasis of OS patients.

In addition, previous studies have shown that HOXB9 is regulated by the Wnt/ $\beta$-catenin/TCF4 pathway $(18,24)$. The Wnt/ $\beta$-catenin signaling plays a critical role in promoting progression and metastasis of multiple cancers (25-27). It has been reported that $\beta$-catenin is the critical effector of the Wnt signaling pathway and played the key role in the transduction of Wnt signal $(28,29)$. It is also known that $\beta$-catenin is targeted to ubiquitin proteasome-mediated degradation (30). FAT10 is a ubiquitin-like protein, and it functions similarly to polyubiquitin as a tag for proteasome targeting (8). In this study, we revealed that silencing FAT10 could decrease the expression of $\beta$-catenin at protein level, but not RAN level, based on which we speculate that FAT10 may regulate $\beta$-catenin at its protein level. This finding suggested a novel possible mechanism of degradation of $\beta$-catenin.

Collectively, we revealed for the first time that FAT10 promoted invasion and metastasis of OS cells. Higher level of FAT10 indicated poor prognosis of OS patients suggesting that FAT10 may be a potential therapeutic target for OS patients.

\section{References}

1. Schwab JH, Springfield DS, Raskin KA, Mankin HJ and Hornicek FJ: What's new in primary bone tumors. J Bone Joint Surg Am 94: 1913-1919, 2012.

2. Cho Y, Jung GH, Chung SH, Kim JY, Choi Y and Kim JD: Long-term survivals of stage IIb osteosarcoma: A 20-year experience in a single institution. Clin Orthop Surg 3: 48-54, 2011. 
3. Lewis VO: What's new in musculoskeletal oncology. J Bone Joint Surg Am 91: 1546-1556, 2009.

4. Marina N, Gebhardt M, Teot L and Gorlick R: Biology and therapeutic advances for pediatric osteosarcoma. Oncologist 9 : 422-441, 2004

5. Wada T, Isu K, Takeda N, Usui M, Ishii S and Yamawaki S: A preliminary report of neoadjuvant chemotherapy NSH-7 study in osteosarcoma: Preoperative salvage chemotherapy based on clinical tumor response and the use of granulocyte colonystimulating factor. Oncology 53: 221-227, 1996.

6. Dikic I, Wakatsuki S and Walters KJ: Ubiquitin-binding domains - from structures to functions. Nat Rev Mol Cell Biol 10: 659-671, 2009.

7. Raasi S, Schmidtke G and Groettrup M: The ubiquitin-like protein FAT10 forms covalent conjugates and induces apoptosis. J Biol Chem 276: 35334-35343, 2001.

8. Hipp MS, Kalveram B, Raasi S, Groettrup M and Schmidtke G: FAT10, a ubiquitin-independent signal for proteasomal degradation. Mol Cell Biol 25: 3483-3491, 2005.

9. Ren J, Wang Y, Gao Y, Mehta SB and Lee CG: FAT10 mediates the effect of TNF- $\alpha$ in inducing chromosomal instability. J Cell Sci 124: 3665-3675, 2011.

10. Merbl Y, Refour P, Patel H, Springer M and Kirschner MW: Profiling of ubiquitin-like modifications reveals features of mitotic control. Cell 152: 1160-1172, 2013.

11. Ji F, Jin X, Jiao CH, Xu QW, Wang ZW and Chen YL: FAT10 level in human gastric cancer and its relation with mutant p53 level, lymph node metastasis and TNM staging. World J Gastroenterol 15: 2228-2233, 2009.

12. Lee CG, Ren J, Cheong IS, Ban KH, Ooi LL, Yong Tan S, Kan A, Nuchprayoon I, Jin R, Lee KH, et al: Expression of the FAT10 gene is highly upregulated in hepatocellular carcinoma and other gastrointestinal and gynecological cancers. Oncogene 22: 2592-2603, 2003.

13. Lukasiak S, Schiller C, Oehlschlaeger P, Schmidtke G, Krause P, Legler DF, Autschbach F, Schirmacher P, Breuhahn K and Groettrup M: Proinflammatory cytokines cause FAT10 upregulation in cancers of liver and colon. Oncogene 27: 6068-6074, 2008

14. Sun GH, Liu YD, Yu G, Li N, Sun X and Yang J: Increased FAT10 expression is related to poor prognosis in pancreatic ductal adenocarcinoma. Tumour Biol 35: 5167-5171, 2014.

15. Yuan J, Tu Y, Mao X, He S, Wang L, Fu G, Zong J and Zhang Y: Increased expression of FAT10 is correlated with progression and prognosis of human glioma. Pathol Oncol Res 18: 833-839, 2012

16. Yuan R, Wang K, Hu J, Yan C, Li M, Yu X, Liu X, Lei J, Guo W, $\mathrm{Wu} \mathrm{L}$, et al: Ubiquitin-like protein FAT10 promotes the invasion and metastasis of hepatocellular carcinoma by modifying $\beta$-catenin degradation. Cancer Res 74: 5287-5300, 2014.
17. Abate-Shen C: Deregulated homeobox gene expression in cancer: Cause or consequence? Nat Rev Cancer 2: 777-785, 2002.

18. Nguyen DX, Chiang AC, Zhang XH, Kim JY, Kris MG, Ladany M, Gerald WL and Massagué J: WNT/TCF signaling through LEF1 and HOXB9 mediates lung adenocarcinoma metastasis. Cell 138: 51-62, 2009.

19. Hayashida T, Takahashi F, Chiba N, Brachtel E, Takahashi M, Godin-Heymann N, Gross KW, Vivanco M, Wijendran V, Shioda T, et al: HOXB9, a gene overexpressed in breast cancer, promotes tumorigenicity and lung metastasis. Proc Natl Acad Sci USA 107: 1100-1105, 2010.

20. Wen M, Kwon Y, Wang Y, Mao JH and Wei G: Elevated expression of UBE2T exhibits oncogenic properties in human prostate cancer. Oncotarget 6: 25226-25239, 2015.

21. Li Y, Guessous F, Johnson EB, Eberhart CG, Li XN, Shu Q, Fan S, Lal B, Laterra J, Schiff D, et al: Functional and molecular interactions between the HGF/c-Met pathway and c-Myc in large-cell medulloblastoma. Lab Invest 88: 98-111, 2008.

22. Gao Y, Theng SS, Zhuo J, Teo WB, Ren J and Lee CG: FAT10, an ubiquitin-like protein, confers malignant properties in nontumorigenic and tumorigenic cells. Carcinogenesis 35: 923-934, 2014.

23. Liu L, Dong Z, Liang J, Cao C, Sun J, Ding Y and Wu D: As an independent prognostic factor, FAT10 promotes hepatitis B virusrelated hepatocellular carcinoma progression via Akt/GSK3 $\beta$ pathway. Oncogene 33: 909-920, 2014.

24. Hatzis P, van der Flier LG, van Driel MA, Guryev V, Nielsen F, Denissov S, Nijman IJ, Koster J, Santo EE, Welboren W, et al: Genome-wide pattern of TCF7L2/TCF4 chromatin occupancy in colorectal cancer cells. Mol Cell Biol 28: 2732-2744, 2008

25 . Miki T, Yasuda SY and Kahn M: Wnt/ $\beta$-catenin signaling in embryonic stem cell self-renewal and somatic cell reprogramming. Stem Cell Rev 7: 836-846, 2011.

26. Lai TY, Su CC, Kuo WW, Yeh YL, Kuo WH, Tsai FJ, Tsai CH, Weng YJ, Huang CY and Chen LM: $\beta$-catenin plays a key role in metastasis of human hepatocellular carcinoma. Oncol Rep 26: 415-422, 2011

27. Lin SY, Xia W, Wang JC, Kwong KY, Spohn B, Wen Y, Pestell RG and Hung MC: Beta-catenin, a novel prognostic marker for breast cancer: Its roles in cyclin D1 expression and cancer progression. Proc Natl Acad Sci USA 97: 4262-4266, 2000.

28. Polakis P: The oncogenic activation of beta-catenin. Curr Opin Genet Dev 9: 15-21, 1999.

29. Rubinfeld B, Robbins P, El-Gamil M, Albert I, Porfiri E and Polakis P: Stabilization of beta-catenin by genetic defects in melanoma cell lines. Science 275: 1790-1792, 1997.

30. Aberle H, Bauer A, Stappert J, Kispert A and Kemler R: betacatenin is a target for the ubiquitin-proteasome pathway. EMBO J 16: 3797-3804, 1997. 